Chapman University

Chapman University Digital Commons

Pharmacy Faculty Articles and Research

School of Pharmacy

$5-2008$

\title{
Nanomaterials and Nanotechnologies: Approaching the Crest of this Big Wave
}

Vuk Uskoković

Chapman University, uskokovi@chapman.edu

Follow this and additional works at: http://digitalcommons.chapman.edu/pharmacy_articles

Part of the Nanotechnology Commons

\section{Recommended Citation}

Uskoković V. Nanomaterials and nanotechnologies: Approaching the crest of this big wave. Curr Nanosci. 2008;4(2):119-129. doi: $10.2174 / 157341308784340903$

This Article is brought to you for free and open access by the School of Pharmacy at Chapman University Digital Commons. It has been accepted for inclusion in Pharmacy Faculty Articles and Research by an authorized administrator of Chapman University Digital Commons. For more information, please contact laughtin@chapman.edu. 


\section{Nanomaterials and Nanotechnologies: Approaching the Crest of this Big \\ Wave}

\section{Comments}

This is a pre-copy-editing, author-produced PDF of an article accepted for publication in Current Nanoscience, volume 4, issue 2, in 2008 following peer review. The definitive publisher-authenticated version is available online at DOI: 10.2174/157341308784340903.

\section{Copyright}

Bentham Science Publishers 


\title{
Nanomaterials and Nanotechnologies: Approaching the Crest of this Big Wave
}

\author{
Vuk Uskoković
}

Jožef Stefan Institute, Advanced Materials Department, Jamova 39, 1000 Ljubljana, Slovenia

\begin{abstract}
Nanomaterials are typically considered as solid physical structures that comprise grain boundaries at the resolution of less than 100 nanometers, whereby nanotechnologies are depicted as dealing with the design of various applications based on employing the former. Some of the essential features of nanomaterials and the scientific approaches to their investigation are discussed in the course of this work. The real reason for the current scientific and technological interest in the physical effects at nano scale is linked with the historic trend of refinement of human knowledge and of the corresponding ability to manipulate with the structural patterns of the Universe. Interesting novel properties of nanomaterials are presented as resulting from the interplay between the surface properties and quantum effects at nano scale. Examples of peculiar combination properties that materials can exhibit with the transition to nanosized form are mentioned, with a particular emphasis on the nanoscopic aggregates of water molecules. Specific challenges tied with the further growth of the field, including the perspectives of functional superstructuring, biomimicry, green chemistry, and the interdisciplinary approach to research, are eventually outlined.
\end{abstract}

\section{The rise and the fundamental aspects of nanosciences}

Nanomaterials are typically considered as solid physical structures that comprise grain boundaries at the resolution of less than 100 nanometers, whereby nanotechnologies are depicted as dealing with the design of various applications based on employing the former ${ }^{1}$.

Whereas nanomaterials have existed since the rise of the human civilization, be it in the form of atmospheric dust particles, lustrous Egyptian ornaments or the earliest tools and machinery ${ }^{2,3}$, 'nanotechnology' is a term coined and introduced into scientific terminology by Japanese engineer Norio Taniguchi. It originally implied a new technology that would go beyond controlling materials and engineering on micro scale ${ }^{4}$. However, the real initiation of nanodreams, that is, engraining limitlessly diverse messages within nanoscale physical patterns, can be traced back to the famous talk that Richard Feynman held at Caltech in late $1959^{5}$. This view of endless possibilities behind the implementation of nanotechnologies as coarse modifiers of the existing material world is nowadays being frequently challenged by the ideas that cohere with the following passage from the thinking of late Richard E. Smalley: 'Much like you cannot make a boy and a girl fall in love with each other simply by pushing them together, you cannot make precise chemistry occur as desired between two molecular objects with simple mechanical motion along a few degrees of freedom in the assembler-fixed frame of reference. Chemistry, like love, is more subtle than that. You need to guide the reactants down a particular reaction coordinate, and this coordinate treads through a many-dimensional hyperspace ${ }^{6}$. And such an idea may mark the grounds of self- 
assembly approaches to the design of nanostructures ${ }^{7}$, as opposing the manipulative methodologies envisaged by the proponents of the classical view of the design and the functional role of nanotechnologies ${ }^{8}$.

The real reason why scientific inquiry increasingly faces physical phenomena on nano scale lies in gradual refinement of human knowledge and of the corresponding ability to manipulate with the structural patterns of the Universe. The historic extrapolation of the current line of progress inevitably points to the exponential growth in the amount of information ${ }^{9}$, whereby the latter could be quantitatively correlated with the amount of 'readable' differences/boundaries ${ }^{10}$. The development of sensitivity and resolution of human tools for discerning and manipulating with the physical matter have naturally led to the descent of human inquiry towards ever smaller phenomena and size scales. It becomes clear that today's nano-hype in scientific society and popular media is, therefore, considerably initiated by large investment funds with a vested interest in, as a matter of fact, ever finer creation of ever subtler technological products in an ever more sensitive relationship between men and Nature.

The peculiar feature of nanomaterials is that, unlike microstructures in which the degree of crystalline order surpasses the degree of disorder in terms of interfacial defects and disorganized grain boundary regions, and unlike amorphous materials in which the crystalline interfacial disorder extends throughout the whole material, this order/disorder ratio is typically in the close range around unity. For example, particles with a few nanometers in size ( $3-5 \mathrm{~nm}$ depending on the particle shape) would possess approximately half of their atoms positioned within the particle surface layers, which are normally of amorphous character. If we also take into account that disrupted atomic bonding typically occurs in the first five layers of atoms at the interface, even larger particles might be considered as comprising this equable order/disorder balance in crystallinity ${ }^{11}$. The interplay between the bulk properties of a material and its interfacial features is responsible for a wide array of new characteristics that arise specifically within its nanoscale forms.

There are two general aspects inherent to the physical chemistry of nanomaterials: practical and fundamental. The very notion of 'materials', in fact, signifies the former aspect, since it denotes only those types of physical matter that were either produced or implemented by the means of pragmatic, artificial design. The practical aspect might be associated with the classical segments in the conceptual framework of materials science: synthesis, characterization, processing, performance and technological application, whereas the fundamental aspect would be limited to the theoretical relationships between the measurements of material structure and the corresponding properties. Fig.1 presents a scheme in which, unlike in the standard textbooks on the subject of materials science ${ }^{12}$, the influence between synthesis, properties, performance and application is presented in a circular manner. Although often ostensibly disconnected, the end aspects of this scheme (design of novel methods of synthesis and the final application forms) are, similar to the fundamental and practical aspects of any science, not unrelated, and should not be, by any means, investigated independently and without reference to each other.

Be that as it may, it has always been a crucial question on the real prospects of this interrelation between the two. The example of DLVO theory from the field of colloid chemistry might be instructive in this sense. Namely, the development of this theory was initiated and supported by the industry in the 1940s, which at that time aimed at 
possessing a general conceptual algorithm for producing stable dispersions of fine particles $^{13}$. However, although developed in a form that has been used as the basis for describing and predicting colloidal phenomena for more than half a century, the synthetic approaches nowadays rely thereupon only in a general and, so to say, 'sketchy' manner. Simply saying, the latter, experimental line of development has gone way forward comparing to the theoretical knowledge base, so that astonishing ultrafine structures are nowadays obtained in laboratories world-wide by employing 'self-assembly' processes which, as their name itself denotes, stand for something formed suddenly and unexpectedly, without any precise underlying design. The prospect of defining the missing links between the theory and synthesis, therefore, presents the first station on our list of major challenges for the contemporary knowledge on the realm of nano.

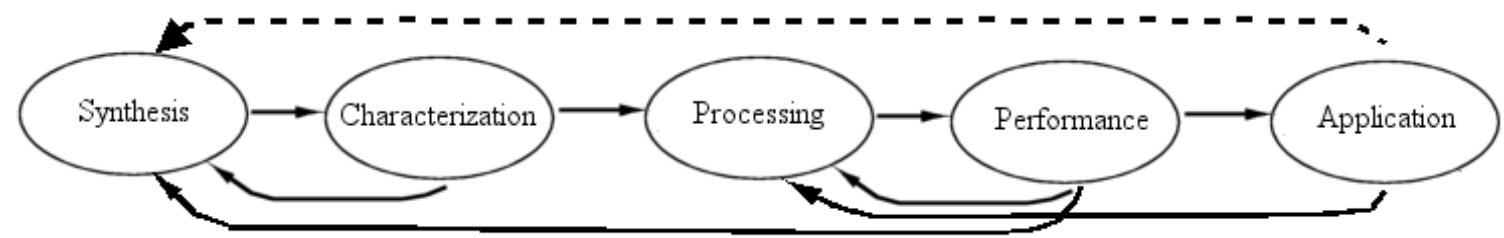

Fig.1. The classical linear relationship between synthesis, characterization, processing, performance and application, transformed into a circular relationship wherein the application forms affect the synthetic and processing design of novel materials and technologies. As an example of how the existing technologies influence the development of the novel ones, recall how engravings in stone, feather quill pens, chalk and blackboard, typing machine and word processing programs all induced specific approaches to writing and communication, which on the other hand were used as media for the construction of blueprints for future writing and communication tools.

\section{Complexities arrived at via extrapolation of micro-to-nano transitions}

The place for one of the most prosperous scientific fields that have recently emerged certainly belongs to the so-called 'nanosciences'. The reason behind the establishment of the whole new field of scientific inquiry that would specifically deal with physicochemical effects that occur on nano spatial scale can be found in enormous complexities that accompany extrapolations of nanomaterial properties from the corresponding chemical structures at either larger, microstructural or smaller, quantum size scales. In fact, nanostructures could be in many cases reasonably depicted as positioned exactly at the interface between the levels of physical interactions governed by the principles of classical and quantum mechanics. Despite the enormous amount of experimentally derived data on micro-to-nano transitions for particular chemical compositions and the corresponding methods of synthesis, there is still no theoretical scheme which would enable the prediction of the behavior of materials in general with their dispersion in the nano range. Hence, new theoretical approaches need to be invoked in describing the physical features of such materials. On the other hand, syntheses of nanomaterials in many cases lead to unexplainable features thereof, preventing the general pursuance of designing approach. All this calls for combined scientific efforts devoted specifically to the investigation of physical phenomena at nano scale.

Two major classes of effects are typically regarded as responsible for these discrepancies that follow extrapolations of materials properties from micro to nano scales: quantum effects and surface effects. In case of the former, confinement effects (i.e. quantization of energy states) induce specific nanosized atomic or molecular 
aggregates to behave as large quantum entities (a.k.a. quantum dots), and obey the probabilistic rules of quantum mechanics. Conjoining precise fabricating control of larger, nanoscale entities with their quantum, discrete physical properties and behavior leads to a wide array of applications, so far mostly in the field of photonics.

In case of the latter, surface effects, increased grain boundary interface leads normally to more pronounced reactivity of the system, sometimes drastically modifying its physicochemical behavior. For example, because liquid phase at nano-dimensions has lower surface energy than a solid with all the facets, edges and corners, solid particles with only a few nanometers in size can frequently display twice lower melting temperature comparing to their corresponding bulk solids ${ }^{14}$. Many inert and non-reactive metals, such as gold, become highly efficient catalysts as the transition to nanoscale order is induced. Increased reactivity of nanoparticles makes them frequently difficult for handling, not only because of their tendency to adsorb contamination, but also because even compositions that are inert and stable in atmospheric conditions may act as pyrophoric and even explosive once they are found in air. Such is the case with nanosized alumina powders that spontaneously combust in air, and may allegedly be used as a rocket fuel despite their well-documented stability in the bulk form ${ }^{15}$. Other metals, including magnesium, zirconium, titanium, tantalum and thorium are in no way different, as they will in nanosized form ignite in air at concentrations at the order of a few tens of grams per cubic meter, caused only by a spark or a hot surface. This is only one of the safety concerns over the upcoming and most probably massive introduction of nanoparticles in technological devices, human households and the environment itself.

Surface and quantum effects can, of course, intermingle in a variety of ways. For example, by reducing the grain size of a material, critical dimensions for specific physical effects might be reached, producing a variety of unexpected properties. Ultra-small capacitors at fine grain boundaries might be thus charged with even single electrons, strongly influencing the subsequent transport of charges through the material ${ }^{16}$. Although electrical resistivity of a conducting material typically becomes lower with increasing the proportion of grain boundaries, it is less known that insulating compounds may become conductive when their constitutive particles are reduced to nanosizes. Furthermore, an aggregate of approximately $10^{4}-10^{6}$ atoms, for instance, displays significantly different behavior comparing to the macroscopic system of identical composition ${ }^{17}$. This hypothetic entity possesses too large number of atoms to allow for extended atomistic simulations by employing quantum properties, such as 'first principles' calculations, and yet it is sufficiently small to enable observation of the melting phenomena as significantly size-dependent.

Concerning the mechanical behavior, it may be mentioned that metals malleable in microstructural forms may come to possess unacceptable levels of creep when their grains are reduced to nanolevel, whereby the formability of typically hard, stiff and brittle ceramics is known to improve with the reduction of grain sizes towards nanoscale. Certain ceramic materials, such as $\mathrm{Y}_{2} \mathrm{O}_{3}$-stabilized $\mathrm{ZrO}_{2}$, liquid-phase sintered $\mathrm{Si}_{3} \mathrm{~N}_{4}$ or even $\mathrm{SiC}$ may, thus, above approximately half the absolute melting point exhibit superplastic behavior ${ }^{18}$. Also, since mechanical failure of materials most frequently takes place through crack migration processes along grain interfaces, significant modifications of strength and toughness mechanisms with the transition from bulk to nanoscale simulations are required in case of the materials that are in nanosized form stronger 
comparing to their bulk counterparts. The classical Hall-Petch relation generally fails to fit experimentally observed hardness vs. grain size dependencies at sufficiently small grain sizes that pertain to the nano range (typically $<10 \mathrm{~nm}$ ), whereas additional phases in complex composite structures frequently change the mechanical properties of the former nanocrystalline matrices in unexpected ways, with often exhibiting sudden slopes in some of the mechanical qualities at only few nanometers wide particle size ranges ${ }^{19}$.

Polygonal particles, fibers, layers, intergranular films, disc-shaped, acicular, lamellar, cylindrical particles or bicontinuous structures may, for instance, reach critical dimensions in only one direction, resulting in a peculiar behavior, often hard to predict by the conventional means ${ }^{20}$. Therefore, due to complex competition between surface and quantum effects as the nanoscale organization in a given material structure is approached, the attempts to model the corresponding phase transitions become seriously hindered. Take the example from the kinetics of solidification. Namely, whereas increased undercooling facilitates nucleation of the solid phase, at the same time it slows down the atomic motion and the diffusion processes, which lowers the transformation rate as well. Furthermore, with an ongoing phase transition, the critical energy barrier is subject to change. However, it seems to be a common nature of scientists in general to base modest and sketchy explanations on single-parameters models and linear relationships, and to elaborate pretentious explanations in terms of complex, dynamic, non-linear and antagonistic relationships within multi-variable models in case of all serious theoretical conceptualizations.

Pronounced surface effects governing the physical behavior of nanomaterials lead to another important consequence. Namely, instead of covalent and ionic forces that act predominantly on microscale, weak forces that among others include van der Waals interaction, hydrogen bonds, chemisorption and surface tension become significantly involved in defining the resulting behavior of a material. The effects of these weak links are in the simplest terms observed in case of the typically disadvantageous phenomenon of agglomeration of particles in the samples with high surface area. As a matter of fact, spontaneous agglomeration effects (which characteristically take place during the final stages of powder synthesis, such as segregation or drying) caused by these weak forces occur in as low packing densities as $1 \%$ of the theoretical. Therefore, due to extensive bonds between individual particles, certain systems, despite being of nanosized nature, behave as microcrystalline systems. Agglomeration effects may also be the source of informal discussions on the topic of when, in fact, a specific material can be endowed with the attribute of nanostructural. On the other hand, despite presenting an undesirable effect in many cases (particularly when individual and well-defined particles are aimed at), these weak forces present a source of delivering a sufficient cohesive strength to a material, improving its mechanical response. Also, these weak forces can be a surprising driving force for a self-assembling association of nanoparticles within multidimensional functional arrays thereof. As a matter of fact, the practical areas of supramolecular chemistry rely exactly on the application of these weak molecular effects for the purpose of templating functional inorganic structures. High selectivity and directionality are typical for molecular bonding that involves these weak links, responsible also for essentially all molecular recognition effects in the biological realm. Anyhow, it has been argued that whereas the traditional field of chemistry has been developed by understanding the effects of covalent, ionic and metallic bonding forces, an extension of 
the same approach to weak intermolecular forces is nowadays suggested as a natural direction for achieving future prosperity within the practical aspects of the field of chemistry $^{21}$. In that sense, we can be reminded of the recent message of George $\mathrm{M}$. Whitesides, one of the 'gurus' in the research of self-assembly phenomena: 'We are at a wonderful time for chemistry. It is, I believe, in the position of physics in the 1910s, just before quantum mechanics made the world impossibly strange, or biology in the 1950s, just before the double helix obliterated the old biology ${ }^{22}$.

It is exactly the unlimited potential of obtainable structures through comparatively simple, eco-efficient experimental settings that has sparked the current scientific interests in self-assembly synthetic methodologies. However, as stated earlier, the very concept of self-assembly implies the existence of physicochemical effects without a precise explanation thereof. In spite of the fact that trial-and-error approaches have been ever since typical for all practical fields of science in general and chemistry in particular, this highly intricate and enigmatic character of self-assembly effects might be, in fact, the major reason behind their attractiveness for the modern chemists. For example, a roomtemperature synthesis of calcium molybdate, one of many ceramics for which hightemperature treatments were previously required to initiate their formation, has recently been reported by a simple precipitation reaction induced by the presence of specific phage peptides, previously eluted in panning experiments on a powder of the targeted composition $^{23}$. Still, significant discrepancies in terms of the powder properties were noticed upon the mildest modifications of the peptide structures. On the other hand, propositions are made to reduce the function of complex molecular structures down to simpler analytical or numerical terms, such as in the cases of identification of the selectivity of protein interactions by the means of various spectral and space-frequency analyses $^{24}$. Such approaches could yield information on the key active amino acid sequences within proteins, thus facilitating the efficiency of their application. But consequently, there are also prospects that through such approaches the actual, easily visualized and mechanistic models used to explain the self-assembly phenomena (e.g. selective attachments of organic molecules that constrain or favor certain crystal growth directions) will eventually cede their place to abstract explanatory models, in a way similar to what happened to the picture of atom at the turn of the $20^{\text {th }}$ Century. Thereupon, it seems that the common fate of the scientific progress in general is that each simplification inevitably implies a parallel rise in the inherent complexity. On the other hand, it is worth noting that all the inestimable complexities in the world of science necessarily hide some elegant simplicity within as well.

All in all, the intercrossing of surface and quantum effects at nano level implies the quest for novel methods and languages to probe and describe the resulting processes. In fact, it would be really interesting to follow how the 'language' of the models used to explain the physical phenomena at nano scale would evolve. Already, as a consequence of inapplicability of both statistical many-body theories and the language of classical physics used for describing macroscopic and microscopic processes on one side, and a similarly difficult and discrepant application of quantum calculations on the other, the current language used to describe and explain phenomena on nanoscopic and mesoscopic scales finds itself on an awkward 'middle ground'. Developing a unique and pragmatic character thereof by blending the quantum and classical explanatory approaches presents a significant challenge on part of the contemporary nanoscientists. 
Now, these complexities faced in the investigation of nanoscale effects leave their trace in the domain of economy of nanomaterials and nanotechnologies as well. That is, despite the fact that the US federal funding for research of nanotechnologies quadrupled from about $\$ 270$ million in 2000 to $\$ 1.08$ billion in 2006, and that the mentions of 'nanotechnology' in the popular press have been growing exponentially (more than doubling in 2004 alone), the predictions of the future prospects of the proliferation of nanotechnologies seem to have been overoptimistic in a way. Namely, in 2004, the US National Science Foundation had predicted that a global market for nanotech products and services could top $\$ 1$ trillion by $2010^{25}$, whereby two years later the predictions from the same institution shifted the given target to 2015. Other comprehensive estimates for the upcoming global nanotechnology market were even more modest ${ }^{26}$, predicting an average annual growth rate of only $19.1 \%$ between 2006 and 2011, reaching US\$25 billion in 2011. Although nanomaterials could be considered as precursors for the development of nanotools and nanodevices, they still comprise about $87 \%$ of the global nanotechnology market (nanotools accounted for $10 \%$, and nanodevices for the remaining $3 \%$, whereby it is worth recalling that there are still no strict definitions of these, which would prevent, for example, including brand new cars into value only because there were nanoparticles in their paintwork ${ }^{27}$ ). By creating unrealistic expectations for nanotechnologies, the contemporary nano-hype that surrounds the attempts to cash in or attract funds by the means of mere reference to 'nano', may be seen as one of the causes behind these inflated predictions. As marketing opportunities of nanoproducts are founded upon the rigorous scientific understanding of nanoscale phenomena and the corresponding ability to control and utilize them, the aforementioned stagnations in the development of the latter could be regarded as directly reflecting on the less enthusiastic predictions for the upcoming economy of nanotechnologies.

\section{The example of water}

Water can present a nice example of how a material can possess thoroughly different inherent properties depending on the size of its dispersed atomic or molecular aggregates in the nano range. First of all, the behavior of liquid molecules confined in nanosized spaces or at solid-liquid interfaces in general, due to surface-induced structuring, significantly differs from their behavior within a bulk system ${ }^{28}$. Despite the routinely used assumption that water has bulk properties all the way down to 'contact' with the surrounding phase, it undergoes significant modifications in nanodomain structure, molecular mobility, hydrogen bonding, relaxation processes and the overall solvent characteristics as it approaches foreign surfaces ${ }^{29}$.

It has been, for example, shown that water molecules dispersed in form of nanosized droplets, as in reverse micellar microemulsions ${ }^{30}$, can be divided into three groups based on their mobility. Fourier Transforming Infrared studies have indicated that the water interior of a reverse micelle has a multilayered structure, consisting of interfacial, intermediate and core water regions. The interfacial layer is composed of water molecules that are bounded directly to polar head-groups of the surfactant; the intermediate layer consists of the next few nearest-neighbor water molecules that can exchange their states with the interfacial water; and the core layer is found at the interior

of the water pool and has the properties of the bulk water ${ }^{31}$. Depending on the size of 
reverse micelles, available water may have significantly different solvent properties, ranging from highly structured interiors to free water cores that approximate bulk water solvent characteristics ${ }^{32,33}$.

Although two major types of water structure and reactivity can be distinguished a less dense water region with an open hydrogen-bonded network against hydrophobic surfaces, and a more dense water region with a collapsed hydrogen-bonded network against hydrophilic surfaces - diverse self-association mechanisms can result depending on finely localized solvent properties against hydrophilic and hydrophobic surfaces ${ }^{34}$. It is well-known that water confined within the intracellular matrix possesses nanoscopic boundaries, which provides cytoplasmatic medium with the properties of a colloid gel instead of an ordinary solution. Exceptional water-retaining properties of the cellular gel matrix are in agreement with its large inherent concentration of interfaces between water and intracellular proteins, polysaccharides, nucleic acids and lipid membranes. In that sense, it is worth recalling that in this, nanosized form, 'water acts at the contact between biological molecules, not just separating them, but imparting information among them', as Martin Chaplin from London South Bank University points out. 'In an aqueous environment, all the molecules are able to feel the structure of all the other molecules that are present, so they can work as a whole rather than as individuals', Chaplin further argues. Hydrogen bonding in water is, in fact, one of the fundamental biochemical interactions involved in enzyme catalysis, protein folding, DNA base-pairing, respiration and photosynthesis. It also comprises highly delocalized protons, indicating that quantum delocalization effects may play central role in biological interactions of water at the nano level $^{35}$. 'Water behaves very differently from other small molecules. If you want something else with similar properties, you would end up with something much bigger and more complex, and then you would lose the advantages that water has in being small’, says Jill Granger from Sweet Briar College in Virginia ${ }^{36}$.

Different water structures may, then, dissolve different amounts of gases, which can drastically influence the intrinsic reaction pathways, particularly in the cases where oxidation or reduction reactions by means of dissolved gases comprise crucial steps in the preparation procedures ${ }^{37}$. In order to understand the interfacial interaction of water droplets with the surrounding phases, it has been mentioned that required are interdisciplinary research efforts that would conjoin the fields of physical chemistry (that refers to electrostatic and van der Waals interactions), surface chemistry (oriented towards the phenomena arising out of the effects of interfacial free energies and surface structures), statistical dynamics (useful in explaining the effect of pinning of contact lines and the transitions among wetting morphologies), and fluid dynamics (related to phenomena of liquid spreading and capillary flow) ${ }^{38}$. As a result, water and correspondingly any other chemical composition in nanosized form could not be treated as continuous media, irrespective of the intrinsic and interfacial interactions that occur on an ultrafine scale.

\section{Future challenges and the directions for growth}

One of the natural questions that arise from the descent of scientific inquiry towards investigation and control of physical phenomena occurring on ever smaller size scales is: How far could we go? Heisenberg's uncertainty principle outlines the ultimate 
limit of physical measurements at the order of magnitude of Planck's constant, but many other effects constrain the design and application of microscopic mechanical effects at the nano scale. For example, environmental gas particles that continuously adsorb and desorb to and from the surface of a nanoscopic device could induce weight fluctuations which would in proportion to size and sensitivity of the given device prove to be significant constraint to its function ${ }^{39}$. Also, ubiquitous, random thermal fluctuations impose a "noise floor" below which it is impossible to discern signals from the background noise. The fundamental barrier to a limitless continuation of Moore's law is inherently related to this inability to constrain the influence of random thermal fluctuations on the computational performance of electronic devices.

Every form of measurement and communication necessarily perturbs the measured and communicated system. It is known that all microscopic analyses provide images that form as an intersection of the properties and settings of the given apparatus and the properties of the measured system. For example, atomic force microscopy (AFM), one of the most powerful techniques for visualization of the structure of materials on atomic scale, provides images that are actually convolutions of the AFM tip shape and the surface morphology. The essential question in this context is until what extent the science would be able to increase the resolution of its interference with ultrafine physical effects before starting to observe only the reflections of the measuring devices and the questions that underlie scientific inquiry. The challenges of extending the actual limits to practical scientific and technological capability of controlling the physical processes on small scales and, so to say, receiving the empirical answers from Nature, are important as ever in the field of nanoscience.

The future prospect in the development of the fields of nanoscience and nanotechnologies is in certain extent associated with the blending of various experimental techniques, synthetic approaches and analytic procedures for the sake of building more complex and thorough representations of the phenomena that take place on the nano scale. The merging of bioengineering and nanostructural materials will, for example, inevitably take place, but how prominent and pervasive these new bionanotechnologies will be is still a question. However, one thing is for certain. Namely, biological creatures in general and human beings in particular can be regarded as the most sophisticated bionanomachines of the current era of the life on Earth ${ }^{40}$. And these machines were formed not by precise external design, but through evolutionary trial-and-error approaches coupled with self-assembly mechanisms that have guided the biosynthetic processes. On top of knowing this, an implementation of biomimetic, self-organizing methodologies in the development of advanced methods of synthesis apparently needs to be considered.

Interdisciplinarity in many aspects is, therefore, the next big thing that everyone mentions, but not too many scientists are willing to seriously bite into it. However, a successful development of nanotechnologies obviously requires well-coordinated efforts of numerous scientific fields, including physics, chemistry, biology, ecology and environmental science. For example, a research center at Cambridge University has recently appointed its first lab-based social scientist in order to bring the reflection of social values and needs into real-time research supervision and practice ${ }^{41}$. Then, there is no wonder that Rockefeller University, not divided to individual departments with the purpose of fostering open communication between various specialists within, has been 
recently selected as the most successful scientific institution in the world in terms of the percentage of originated scientific breakthroughs ${ }^{42}$. However, living in the era in which, in the words of the Editor of 'Nature', partially hypothetic papers written in a fashion in which Crick and Watson announced their discovery of the structure of DNA and linked it with its proposed role in the genetic development would be regularly denied publication had the manuscript been peer-reviewed (let alone the recent cases of notable publishers routinely refusing slightly, for fun modified timeless masterpiece books of famous authors), seems to be asking for something changed. It is necessary to lean on the productive approaches and paradigms in pursuing scientific method, but an openness to adopt novel patterns of research, particularly the ones that deal with the discussion of scientific efforts from philosophical, humanistic and artistic perspectives, has to be achieved.

Arts have a great power to inspire, and as such they may be regarded as a perfect complementarity to the pragmatism of scientific endeavors in general. Serious scientific literature, therefore, ought to be receptive for discourses that stretch between the two aspects of human creativity. Scientific method should not be envisaged with the dose of inspiration only in popular books that in mostly anecdotal manner represent the historical events, but ought to be also linked in many ways with other, although in this context rarely seriously mentioned areas of human inquiry about Nature, including philosophy, sociology, environmental science, ethics and aesthetics. In the end, as most investments of scientific projects, including the ones of nanotechnological significance, come from public sources $^{43}$, a diversity of social perspectives has to be included in the R\&D efforts in the area of nanomaterials and their application. However, recall that National Science Foundation of the United States spent $\$ 8$ million on nanotech projects, and did not fund a single project focused on societal implications of nanomaterials and nanotechnologies, whereby the percentage of the citations in scientific databases on the latter subject continually falls in relation to the overall number of citations on nanotechnologies ${ }^{44}$.

Some other questions of both aesthetical and pragmatic relevancy may be also mentioned. For example, it seems that we live in the synthetic era in which an obsession with superficially attractive systems, in terms of uniform and highly symmetrical arrangement of structural boundaries, is fostered by the peer-review selection procedures. However, the fascination with symmetry should present only the first step towards achieving both aesthetical and pragmatic wonder in front of the strange interplay between symmetry and asymmetry, typical for all aspects of biological forms and processes. For example, it is known that the topography of biological crystals exhibits surface irregularities of the order of the size of single unit cells for the given crystal structure ${ }^{45}$. Uniformity does not exist in natural reproduction, and the materials design in the coming era should partly adopt this attitude of fabrication of structures that will be unique, irreproducible and yet reliable upon application, similar to biological creatures. In spite of the current fascinations with uniform spherical particles, it is known that spheres do not appear as suitable structural units for the formation of three-dimensional supramolecular scaffolds. Comparing to the isotropic features of spherical particles that naturally promote their preferential aggregation into close packing, higher levels of structural complexity and informational capacity would be introduced with a shift towards particles anisotropic in shape, texture and physical properties ${ }^{46}$. In that sense, we should also be reminded how numerous investigations on animal cell cultures have resulted in general observations that 
comparing to cancerous cell cultures, typified by their inherently cloned nature permeated by uniform pathways of genetic, epigenetic and metabolic expressions from cell to cell and naturally implied relatively high levels of reproducibility and predictability of experimental interferences therewith, healthy cell cultures are normally characterized by widely modified biochemical properties among individual cells and the high corresponding levels of unreliability, irreproducibility and unpredictability of experimental settings and results based on their utilization. However, what would be the right way to produce perfections through imperfections, as Nature does? Maybe the beginnings in pursuing this line of development would be reflected on investigations of the materials with fractal ${ }^{47}$, fuzzy ${ }^{48}$ or hierarchical internal patterns that emerge novel properties at each organizational complexity level ${ }^{49}$. Or in the words of Jean-Marie Lehn, 'In the context of the 'big' problems challenging science, where physics addresses the origin and laws of the universe, and biology those of life, chemistry may claim to provide the means for unraveling the progressive evolution towards complex matter by uncovering the processes that underlie self-organization, and for implementing the knowledge thus acquired to create novel expressions of complex matter'. On the other hand, as the production of materials with uniform internal patterns increases the structural diversity of the planetary matter, its further progress should be undoubtedly cultivated. Extraordinarily uniform systems nowadays present novel and fascinating design achievements that result in perfectly reproducible properties and performances of the obtained materials.

However, not only are the methods for fabrication of nanoscale powders normally a lot more expensive comparing to their microstructural counterparts, but the ecological costs for their production often follow hand-in-hand. As a consequence of finer intrinsic organization, the class of potentially attainable nanomaterials and nanodevices is wider than the set of all achievable bulk materials (recall in this sense how biological uptake of a given chemical significantly depends on its crystallinity and particle size ${ }^{50,51,52}$ ), which implies that broad regulatory generalizations should cede their place to detailed toxicological and environmental impact assessments of the specific nanoproducts in plan. Neither a moratorium on the release of novel nanoparticles nor clinging to an old set of existing regulations and the idea that "a lack of evidence equals the evidence of lacking" present the right attitudes, but a mutually accentuating balance between development and innovation on one side and regulative precaution on the other ought to be achieved. The standardization of nanoproducts will, therefore, most probably present a long process, rather than an abrupt event ${ }^{53}$.

Another inevitable future direction of progress, therefore, lies in the inherent association of ecological principles within every productive phase of materials science, depicted in Fig.1. That is, it is not enough anymore to recycle materials after their functional life cycles in the frame of given devices is over, but the use of sustainable and eco-efficient methods for fabrication should be implemented in the synthesis stages at the very first place. Considering the fact that there is more than a million new chemical compounds reported each year and close to a thousand new ones being added to the commercial domain each year (besides over 70,000 already existing), without any required training in toxicology or environmental sciences for the doctoral chemists, it is clear that increasing ecological awareness regarding chemical life cycles would present a key component of 'greening' education in chemistry of the coming era ${ }^{54}$. The principles 
of 'green chemistry'55,56,57 should be unavoidably considered as the basics for any synthetic and processing approaches in the chemistry of nanomaterials as well. In that sense, the immediate toxic effects of nanoparticles on biological creatures and the destructive potentials of disobeying nanobots may be regarded as only superficial and anthropomorphic threats, whereas long-term effects that in addition include the problems of ecological sustainability, high-tech economics, intellectual property, social equality and transfer of technologies, should be given a prime interest. The targets of reaching ultimate, zero-waste eco-friendliness would promote an important shift from quantitative, end-of-pipe solutions to implementation of qualitative chemical modifications at the initial, design stages. This would, on the other hand, open endless novel challenges and opportunities for an eco-friendly chemical research.

\section{Conclusion}

As we could have seen from this short discourse, the research of nanomaterials and their technological applications has been taking place for a considerable amount of time, but the real fruits of this work are obviously yet to emerge. As in the case of many other preceding technologies, the scopes of potential perils and benefits seem to neatly balance each other, and it is not through ignoring one or the other, but through constantly referring to and reflecting from each other that this new science will have a chance to become a prosperous and eco-friendly scientific and practical field without antecedence in the history of human creativity. Permanently challenging circumstances in this field apparently coincide with the fact that science in general can be considered as a miraculous adventure of human mind. In any case, an interdisciplinary organization of the research and industrial development of nanoscale physical effects would be inevitably required for their successful implementation in the ecological and social niches.

And finally, we should not stop seeing the relationship between nanosciences and the less referred aspects of human creativity, including epistemology, ethics and aesthetics. In that sense, a short anecdote may be mentioned. Namely, there is a cartoon where an astronomer's wife welcomes a guest to their home in which the astronomer sits engaged in looking through his telescope, commenting: 'Well, as you see, my husband lives in a small, small world of his own'.

Investigations of nanoscale phenomena from a wide array of perspectives offer a similar insight. That is, the focus on seemingly small and negligible features that stand at the interface between quantum effects and macroscopic physical behavior may open the doors to see endlessly permeating features of the physical reality as reflected in all of its minute details. Furthermore, learning to control these nanoscale physical effects will have crucial influence on the upcoming development of advanced, ultrafine technologies. For there is no doubt that nanomaterials will soon come to emanate as essential, dominant and all-pervasive ingredients of the electronically miniaturized world of ours. Let us only hope that in such a world, the qualities of being great and might in being humble, diligent and small, which have comprised the implicit cognitive message of scientific endeavors in general, would be fostered both at the levels of science and technologies and of human-to-human communications. Only then the prophecy of that lovely catchword that says 'hey, small is beautiful' would become crystal clear. 


\section{References}

\footnotetext{
${ }^{1}$ V. Uskoković - "Nanotechnologies: What We Do Not Know”, Technology in Society 29 (1) 43 - 61 (2007).

${ }^{2}$ M. F. Hochella Jr. - “Nanoscience and Technology: The Next Revolution in the Earth Sciences”, Earth and Planetary Science Letters 203, 593 - 605 (2002).

${ }^{3}$ J. P. Arantegui, A. Larrea - "The Secret of Early Nanomaterials is Revealed, Thanks to Transmission Electron Microscopy”, Trends in Analytical Chemistry 22 (5) 327 - 329 (2003).
}

${ }^{4}$ N. Taniguchi - “On the Basic Concept of 'NanoTechnology'“, Proc. Intl. Conf. Prod. Eng. Tokyo, Part II, Japan Society of Precision Engineering, Tokyo (1974).

${ }^{5}$ R. Feynman - “There’s a Plenty of Room at the Bottom: An Invitation to Enter a New Field of Physics”, Lecture at the California Institute of Technology (December 29, 1959).

${ }^{6}$ R. Baum - “Nanotechnology: Drexler and Smalley Make the Case For and Against 'Molecular Assemblers'“, Chemical \& Engineering News 81 (48) (December 1, 2003).

${ }^{7}$ G. Whitesides, B. Grzybowski - “Self-Assembly at All Scales”, Science 295, 2418 - 2421 (2002).

${ }^{8}$ K. E. Drexler - "Engines of Creation: the Coming Era of Nanotechnology”, Anchor, New York, NY (1986).

${ }^{9}$ R. Kurzweil - “Human 2.0.”, NewScientist 32 - 37 (24 September 2005).

${ }^{10}$ G. Bateson - “Mind and Nature: A Necessary Unity”, Hampton Press, Cresskill, NJ (2003).

${ }^{11}$ R. M. German - "Powder Metallurgy and Particulate Materials Processing”, Metal Powder Industries Federation, Princeton, NJ (2005).

${ }^{12}$ W. D. Callister Jr. - "Materials Science and Engineering: An Introduction”, Fifth Edition, John Wiley \& Sons, New York, NY (2000).

13 A. Blume, Th. Zemb - "Self-Assembly: Weak Molecular Forces at Work for Building Mesoscopic Architectures”, Current Opinion in Colloid and Interface Science 7, 66 - 68 (2002).

${ }^{14}$ Y. Yin, P. Alivisatos - “Colloidal Nanocrystal Synthesis and the Organic-Inorganic Interface”, Nature 437, $664-670$ (2005).

${ }^{15}$ ETC Group - “A Tiny Primer on Nano-Scale Technologies and 'The Little Bang Theory'“, Action Group on Erosion, Technology and Concentration, Winnipeg, Canada (June 2005); retrieved from www.etcgroup.org/upload/publication/55/01/tinyprimer_english.pdf.

${ }^{16}$ A. S. Edelstein, J. S. Murday, B. B. Rath - “Challenges in Nanomaterials Design”, Progress in Materials Scence 42, 5 - 21 (1997).

${ }^{17}$ P. W. Sutter, E. A. Sutter - "Dispensing and Surface-Induced Crystallization of Zeptolitre Liquid MetalAlloy Drops”, Nature Materials 6 (5) 363 - 366 (2007). 
18 F. Wakai - “Grain Boundary Dynamics in Nanograin Superplasticity, Grain Growth and Sintering”, Proceedings of the Materials Science and Technology (MS\&T) 2006: Materials and Systems 2, 615 - 624 (2006).

19 C. C. Koch, R. O. Scattergood, K. L. Murty - "Mechanical Behavior of Multiphase Nanocrystalline Materials”, Proceedings of the Materials Science and Technology (MS\&T) 2006: Materials and Systems 2, $347-356$ (2006).

${ }^{20}$ R. D. Shull - “Nanomagnetism: A New Materials Frontier”, Proceedings of the Materials Science and Technology (MS\&T) 2006: Materials and Systems 2, 323 - 329 (2006).

${ }^{21}$ W. M. Tolles - “Self-Assembled Materials”, MRS Bulletin 25 (10) 36 - 38 (2000).

${ }^{22}$ G. M. Whitesides - “Revolutions in Chemistry”, Chemical \& Engineering News 12 - 17 (March 26, 2007).

${ }^{23}$ G. Ahmad, M. B. Dickerson, B. C. Church, Y. Cai, S. E. Jones, R. R. Naik, J. S. King, C. J. Summers, N. Kröger, K. H. Sandhage - "Rapid, Room-Temperature Formation of Crystalline Calcium Molybdate Phosphor Microparticles via Peptide-Induced Precipitation”, Advanced Materials 18, 1759 - 1763 (2006).

${ }^{24}$ I. Ćosić - “The Resonant Recognition Model of Macromolecular Bioactivity: Theory and Applications”, Birkhauser Verlag, Basel, Switzerland (1997).

${ }^{25}$ ETC Group - “Nanotech's 'Second Nature' Patents” (June 2005); retrieved from http://www.etcgroup.org.

${ }^{26}$ Business Communications Company Inc. Report - "Nanotechnology: A Realistic Market Assessment”, BCC00065 (July 2006).

${ }^{27}$ Cientifica - “Nano Market Methodologies” (May 2004), retrieved from http://cientifica.eu.

${ }^{28}$ K. Kurihara - "Nanostructuring of Liquids at Solid-Liquid Interfaces", Progress in Colloid and Polymer Science 121, 49 - 56 (2002).

29 V. M. Gun’ko, V. V. Turov, V. M. Bogatyrev, V. I. Zarko, R. Leboda, E. V. Goncharuk, A. A. Novza, A. V. Turov, A. A. Chuiko - "Unusual Properties of Water at Hydrophilic/Hydrophobic Interfaces", Advances in Colloid and Interface Science 118, 125 - 172 (2005).

${ }^{30}$ V. Uskoković, M. Drofenik - "Synthesis of Materials within Reverse Micelles”, Surface Review and Letters 12 (2) 239 - 277 (2005).

${ }^{31}$ Q. Zhong, D. A. Steinhurst, E. E. Carpenter, J. C. Owrutsky - "Fourier Transform Infrared Spectroscopy of Azide Ion in Reverse Micelles”, Langmuir 18, 7401 - 7408 (2002).

32 J. C. Linehan, J. L. Fulton, R. M. Bean - "Process of Forming Compounds Using Reverse Micelle or Reverse Microemulsion Systems”, US Patent 5,770,172 (1998).

${ }^{33}$ R. Zana, J. Lang - “Dynamics of Microemulsions”, in Microemulsions: Structure and Dynamics, edited by S. E. Friberg and P. Bothorel, Boca Raton: CRC Press, pp. 153 - 172 (1987).

34 E. A. Vogler - "Structure and Reactivity of Water at Biomaterial Surfaces”, Advances in Colloid and Interface Science 74, 69 - 117 (1998). 
${ }^{35}$ J. McFadden - "Quantum Mechanics and Biology”, in Quantum: A Guide for the Perplexed, edited by J. Al-Khalili, Weidenfeld \& Nicolson, London (2003).

${ }^{36}$ N. Angier - “Small, but Mighty: That Molecule Called Water”, The New York Times (July 23, 2007).

${ }^{37}$ V. Uskoković, M. Drofenik - “A Mechanism for the Formation of Nanostructured NiZn Ferrites via a Microemulsion-Assisted Precipitation Method”, Colloids and Surfaces A: Physicochemical and Engineering Aspects 266, 168 - 174 (2005).

${ }^{38}$ Y. Xia, D. Qin, Y. Yin - “Surface Patterning and its Application in Wetting/Dewetting Studies”, Current Opinion in Colloid \& Interface Science 6, 54 - 56 (2001).

${ }^{39}$ M. Roukes - “Plenty of Room Indeed”, Scientific American 285 (3) 43 - 47 (2001).

${ }^{40}$ A. J. I. Vontz, V. Vesna - “In the Future, Every Molecule Will Have Its 15 Minutes of Fame”, Los Angeles Times (August 31, 2003).

${ }^{41}$ J. Wilsdon - "The Politics of Small Things: Nanotechnology, Risk, and Uncertainty”, IEEE Technology and Society Magazine 16 - 21 (Winter 2004).

${ }^{42}$ R. J. Hollingsworth - "High Cognitive Complexity and the Making of Major Scientific Discoveries", in Knowledge, Communication and Creativity, edited by A. Sales and M. Fournier (2007).

${ }^{43}$ Editorial - "Hidden Treasures”, Nature Materials 6 (5) 319 (2007).

${ }^{44}$ A. Mnyusiwalla, A. S. Daar, P. A. Singer - “'Mind the Gap': Science and Ethics in Nanotechnology”, Nanotechnology 14, R9 - R13 (2003).

${ }^{45}$ J. Kirkham, S. J. Brookes, R. C. Shore, S. R. Wood, D. A. Smith, J. Zhang, H. Chen, C. Robinson "Physico-Chemical Properties of Crystal Surfaces in Matrix-Mineral Interactions During Mammalian Biomineralisation”, Current Opinion in Colloid \& Interface Science 7, 124 - 132 (2002).

${ }^{46}$ S. Mann - "Materials that Naturally Assemble Themselves", Chemical Communications (Camb) 1, 1 - 4 (2004).

${ }^{47}$ R. Villegas, A. Redjaimia, M. Confente, M. T. Perrot-Simonetta - "On the Fractal Nature of Acicular Ferrite, and V(C,N) Precipitation in Medium Carbon Microalloyed Steels”, Materials Science and Technology (MS\&T) 2006: Materials and Systems 2, 401 - 410 (2006).

${ }^{48}$ G. Decher - "Fuzzy Nanoassemblies: Toward Layered Polymeric Multicomposites”, Science 277 (5330), 1232 - 1237 (1997).

${ }^{49}$ J.-M. Lehn - "From Supramolecular Chemistry towards Constitutional Dynamic Chemistry and Adaptive Chemistry”, Chemical Society Reviews 36, 151 - 160 (2007).

${ }^{50}$ R. F. Service - “Nanomaterials Show Signs of Toxicity”, Science 300, 243 (2003).

${ }^{51}$ L. K. Limbach, Y. Li, R. N. Grass, T. J. Brunner, M. A. Hintermann, M. Muller, D. Gunther, W. J. Stark - "Oxide Nanoparticles Uptake in Human Lung Fibroblasts: Effects of Particle Size, Agglomeration, and Diffusion at Low Concentrations”, Environmental Science \& Technology 39 (23) 9370 - 9376 (2005).

${ }^{52}$ S. D. Škapin, E. Matijević - "Preparation and Coating of Finely Dispersed Drugs 4. Loratadine and Danazol”, Journal of Colloid and Interface Science 272, 90 - 98 (2004). 
${ }^{53}$ G. H. Reynolds - "Environmental Regulation of Nanotechnology: Some Preliminary Observations”, Environmental Law Reporter 31 (6), 10681 - 10688 (2001).

${ }^{54}$ J. C. Warner, A. S. Cannon, K. M. Dye - “Green Chemistry”, Environmental Impact Assessment Review 24, 775 - 799 (2004).

${ }^{55}$ D. Anderson, J. L. Anthony, A. Chanda, G. Denison, M. Drolet, D. Fort, M. Joselevich, J. R. Whitfield "A New Horizon for Future Scientists: Students Voices from the Pan-American Advanced Studies Institute on Green Chemistry”, Green Chemistry 6, G5 - 9 (2004).

${ }^{56}$ J. H. Clark - “Green Chemistry: Today (and Tomorrow)”, Green Chemistry 8, 17 - 21 (2006).

${ }^{57}$ J. O. Metzger - “Agenda 21 as a Guide for Green Chemistry Research and a Sustainable Future”, Green Chemistry 6, G15 - 16 (2004). 